Artículo de reflexión no derivado de investigación

\title{
Análisis de la Política Nacional de Competitividad y Productividad (2008-2014): oportunidades para el sector apícola colombiano
}

\author{
Daniel Fernando Rincón Yara ${ }^{1 *}$ ¿zootecnista, Est. Maestría
}

Fecha correspondencia: Recibido: 6 de febrero de 2019. Aceptado: 12 de junio de 2019.

Forma de citar:

Rincón Yara, DF. Análisis de la Política Nacional de Competitividad y Productividad (2008-2014): oportunidades para el sector apícola colombiano. Rev. CES Med. Zootec. 2019; Vol 14 (2): 87-107.

Open access

(c) Copyright

Creative commons

Éthics of publications

Peer review

Open Journal System

DOl: http://dx.doi.org/10.21615/

cesmvz.14.2.8

ISSN 1900-9607

Filiación:

1 Estudiante de Maestría en Producción Animal, Línea de investigación en Gestión Empresarial en Sistemas Pecuarios Grupo de investigación en Gestión en Empresas Pecuarias -Gigep. Facultad de Medicina Veterinaria y de Zootecnia, Universidad Nacional de Colombia sede Bogotá, Bogotá D.C. Colombia.dfrincony@unal.edu.co

Comparte

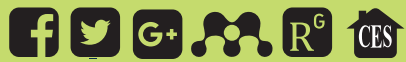

\section{Abstract}

The business environments are delimited by state actions, which are expressed through policy, that in most cases, are unknown by the business managers. This paper to analysis the Política Nacional de Competitividad y Productividad-PNCP, a Colombian competitiveness policy between 2008 and 2014, to identify opportunities for the beekeeping sector. A specific action Plan of the PNCP influences to agricultural sector, inside it, three of the four strategic objective could to offer opportunities for the beekeeping sector, however, the beekeepers trade associations must to cuantificate the effect of this.

Keywords: actions plans, business competitiveness, business environment.

\section{Resumen}

Los entornos en los que las empresas desarrollan sus actividades están demarcados por las acciones estatales que se materializan a partir de los planes de acción de políticas públicas, que en la mayoría de los casos no son conocidos por parte de las directivas de las empresas. El objetivo de este trabajo es analizar la Política Nacional de Competitividad y Productividad entre los años 2008 y 2014 para identificar posibles oportunidades para el sector apícola colombiano. De los quince planes de acción de dicha Política Pública, uno atiende el sector agropecuario y dentro del mismo, tres de los cuatro ejes estratégicos que lo desarrollan ofrecen acciones gubernamentales que representan oportunidades para el sector apícola colombiano, especialmente para el eslabón primario de la cadena de producción. A pesar de ello, el impacto de los mismos debe ser cuantificado por las organizaciones gremiales del sector apícola colombiano.

Palabras clave: competitividad empresarial, entorno empresarial, planes de acción.

\section{Introducción}

Para el año 2008, el sector apícola colombiano firmó el Acuerdo Sectorial de competitividad de la Cadena Productiva de las Abejas y la Apicultura (CPAA), el cual tiene como objetivo promover el mejoramiento de la gestión empresarial, la rentabilidad y la asociatividad y cuyo objeto es mejorar la productividad y la competitividad de la Cadena Productiva con miras al desarrollo integral del sector ${ }^{16}$. 
En dicho Acuerdo tanto el Gobierno Nacional como el sector privado se comprometen a adelantar y adoptar acciones para aumentar la producción, la organización, la capacitación, el nivel tecnológico, la investigación, la calidad, la productividad, el consumo, las exportaciones, la modernización de la industria apícola nacional y regional y toda acción que permita impulsar la competitividad de la apicultura colombiana. Lo anterior, conllevará a mejorar continuamente el nivel de vida de los actores de todos los eslabones de la cadena ${ }^{16}$.

Este Acuerdo se inscribe en el conjunto de reglas institucionales que tiene el país sobre el tema. Dentro de dicho conjunto resalta el documento Conpes No. 3527 de 2008, donde se formula la Política Nacional de Competitividad y Productividad (PNCP), cuyo objetivo es lograr la trasformación productiva del país, a través de la puesta en marcha de 15 planes de acción, que abarcan la mayor cantidad de actividades económicas del país ${ }^{11}$.

Según Vega Almeida (2006), la interacción organización-entorno es constante y dinámica y por consiguiente los resultados obtenidos por la organización estan condicionados por la influencia de factores exógenos (culturales, tecnológicos, educacionales, políticos, legales, sociales y económicos) ${ }^{21}$, por lo que al considerar el objeto y los planes de acción de la PNCP, se hace necesario determinar la influencia de los mismos sobre las organizaciones de productores apicolas en Colombia.

Cabe resaltar que no todas las políticas públicas afectan de similar forma a los sectores económicos, dada la naturaleza de las mismas. Así, mientras políticas sectoriales o verticales están diseñadas especificamente para resolver problemas de un sector económico concreto, las políticas transversales u horizontales son instrumentos que atienden problemas comunes o generalizados en todos los sectores económicos. La Politica Nacional de Competitividad y Productividad es un ejemplo clásico de una política horizontal.

El objetivo del presente trabajo es describir algunos componentes de la PNCP, entre los años 2008 y 2014, a través de la aplicación de la metodología de análisis de política pública propuesta por Knopfel et al (2006), con el fin de identificar elementos que incidan sobre los procesos de mejora de la productividad del eslabón primario de la Cadena Apícola Colombiana. La selección de estos años obedece a la disponibilidad de información en la base de datos del Departamento Nacional de Planeación.

La estructura del mismo está dada por una breve descripción de la prospectiva de la Cadena Productiva de las Abejas y la Apicultura, un bosquejo de la metodología empleada, los resultados de la aplicación de la misma para determinar finalmente los aportes que la PNCP ofrece para el aumento de la competitividad y productividad del sector apícola colombiano.

\section{Panorama del sector apícola colombiano}

En el 2010, la CPAA, estableció las proyecciones de consumo interno percápita promedio, producción y productividad por colmena para el periodo comprendido entre el 2011 y el 2025 para miel y para polen.

El dato sobre consumo interno percapita promedio aparente, se obtiene a partir de dividir la produccion total anual entre la población nacional total anual, y es un indicador no solo del tamaño del mercado, sino también de la aceptación del producto (Tabla 1). 
Tabla 1. Proyecciones de producción, productividad y consumo percápita de los productos principales de la colmena.

\begin{tabular}{|c|c|c|c|}
\hline Temática & 2010 & 2015 & 2025 \\
\hline Producción & $\begin{array}{l}\text { - Miel: } \\
50.000 \text { colmenas } \\
\text { 1.000 ton/año } \\
\text { - Polen } \\
6.000 \text { colmenas } \\
120 \text { ton/año }\end{array}$ & $\begin{array}{l}\text { - Miel: } \\
\text { ( } 10 \% \text { crecimiento anual): } \\
80.526 \text { colmenas } \\
2.013 \text { ton/año } \\
\text { - Polen ( } 5 \% \text { crecimiento } \\
\text { anual): } \\
7.568 \text { colmenas } \\
168 \text { ton/año }\end{array}$ & $\begin{array}{l}\text { - Miel: } \\
\text { ( } 15 \% \text { crecimiento anual): } \\
325.711 \text { colmenas } \\
9.773 \text { ton/año } \\
\text { - Polen: } \\
\text { (8\% crecimiento anual): } \\
16.073 \text { colmenas } \\
402 \text { ton/año }\end{array}$ \\
\hline Productividad & $\begin{array}{l}\text { - Miel: } 20 \mathrm{~g} / \text { colmena/ } \\
\text { año } \\
\text { - Polen: } \\
20 \mathrm{~g} / \text { colmena } / \text { año }\end{array}$ & $\begin{array}{l}\text { - Miel: } 25 \text { kg/colmena/año } \\
\text { - Polen: } \\
38 \text { kg/colmena/año }\end{array}$ & $\begin{array}{l}\text { - Miel: } 30 \text { kg/colmena/año } \\
\text { - Polen: } \\
40 \text { kg/colmena/año }\end{array}$ \\
\hline $\begin{array}{l}\text { Población } \\
\text { colombiana }\end{array}$ & 45.000 .000 & $\begin{array}{l}47.718 .402 \\
\text { (1,18\% crecimiento anual) }\end{array}$ & $\begin{array}{l}53.657 .773 \\
(1,18 \% \text { crecimiento anual) }\end{array}$ \\
\hline $\begin{array}{l}\text { Consumo } \\
\text { percápita } \\
\text { (promedio) }\end{array}$ & $\begin{array}{l}\text { Miel: } 22 \mathrm{gr} \\
\text { Polen: } 2,5 \mathrm{gr}\end{array}$ & $\begin{array}{l}\text { Miel: } 42 \mathrm{gr} \\
\text { Polen: } 3,3 \mathrm{gr}\end{array}$ & $\begin{array}{l}\text { Miel: } 182 \mathrm{gr} \\
\text { Polen: } 6,9 \mathrm{gr}\end{array}$ \\
\hline
\end{tabular}

Fuente: República de Colombia Ministerio de Agricultura y Desarrollo Rural (2011).

Sin embargo y debido a la falta de un sistema de información para el sector, existen serias dudas con respecto a los datos de producción a nivel nacional y de consumo percapita para el caso de la miel, presentados por el Consejo Nacional Apícola, entidad que rige la Cadena Apícola.

Es así como Santamaría (2009), reporta a partir de comunicaciones personales con apicultores reconocidos, que en el País se consumían para ese año, veinte mil (20.000) toneladas de "miel", de las cuales dieciocho mil eran falsificadas (18.000), mil (1.000) eran de produccion nacional y otras mil (1.000) toneladas importadas desde Argentina ${ }^{20}$.

Es decir que para ese año 2009, de acuerdo a esas fuentes, el 90\% del mercado nacional de consumo de miel, eran productos falsamente catalogados como miel de abejas.

Por su parte, el Diagnóstico de la Apicultura Colombiana realizado en el 2006, postula que mínimo el $80 \%$ del total del mercado de miel, está ocupado por productos falsificados ${ }^{4}$.

Ahora bien, si se asume que la producción real de miel en el país (1.000 toneladas) constituye el $20 \%$ del mercado y asumiendo que las toneladas anuales de importación legal sean cero, se tiene que para el año 2010, existian cinco mil (5.000) toneladas de "miel de abejas" en el país, de las cuales cuatro mil (4.000) toneladas (correspondientes al 80\%), es de producto catalogado como "miel de abejas".

Si se hace el cálculo de consumo percápita aparente para los dos datos, se obtiene que de acuerdo con el dato del Diagnostico de la CPAA para el año 2010, el consumo percápita aparente promedio nacional era de 111 gramos/percápita/año de producto catalogado como "miel". Si se realiza el mismo ejercicio con los datos aportados por Santamaría (2009) ese consumo se remonta a 444 gramos/percápita/año. 
A pesar de la gran variabilidad, dispersión y fuentes de información de los datos, queda demostrado que existe un mercado de consumo de miel muy grande, que no ha sido atendido por la producción nacional y es preocupante que cerca del $80 \%$ del mercado nacional sea de un producto falso. Un sector con poca producción, altos niveles de falsificación y pocos niveles de consumo interno, no son buena señal. Y ahí es donde justamente podría entrar la solución por parte del Estado, a través de las políticas públicas.

\section{Modelo de análisis de políticas públicas de Knoepfel et al (2006)}

Para Knoepfel et al (2006), "una política pública es una serie de decisiones o de acciones, intencionalmente coherentes, tomadas por diferentes actores públicos y a veces no públicos, cuyos recursos, nexos institucionales e intereses varían, a fin de resolver de manera puntual un problema políticamente definido como colectivo ${ }^{3}$.

Este conjunto de decisiones y acciones da lugar a actos formales, con un grado de obligatoriedad variable, tendientes a modificar la conducta de grupos sociales que, se supone, originaron el problema colectivo a resolver (grupos-objetivo), en beneficio de grupos sociales que padecen los efectos negativos del problema en cuestión (beneficiarios finales)"3.

Los autores resaltan que en el proceso de formulación de una política pública existen tres elementos clave: los actores, las reglas institucionales y los recursos, elementos que se describen a continuación.

\section{Actores}

Un actor es tanto un individuo (un ministro, un periodista especializado, un diputado), como un grupo de individuos (una oficina, una sección administrativa), o también una persona jurídica (una empresa privada, un sindicato), o incluso un grupo social (agricultores, drogodependientes). Un aspecto importante al momento de definir los actores es su carácter de público o no público (privado) ${ }^{3}$.

De esta forma un actor se considera público cuando sus actos administrativos están vigilados por el derecho público y están sometidos al control gubernamental ${ }^{3}$.

Los autores distinguen dos tipos de actores privados: los "grupos-objetivo", aquellos actores cuya conducta se considera políticamente como la causa directa o indirecta del problema colectivo que la política pública intenta resolver; y los "beneficiarios finales", que son los actores a los que el problema les afecta directamente, sufriendo sus efectos negativos ${ }^{3}$.

Por último, hay un tercer grupo de actores, también dentro de la categoría de privados, llamados "grupos terceros" quienes ven modificada su situación de manera permanente, positiva o negativamente, por acción de la política pública, sin que esta haya sido destinada para ellos de manera puntual ${ }^{3}$.

Finalmente, los autores plantean la existencia de un "triángulo de base" de una política pública constituido por las autoridades político administrativas (actores públicos), los grupos objetivo y los beneficiarios finales (actores privados) ${ }^{3}$. La construcción de este triángulo se verá mejor explicada al momento de aplicar la metodología al caso de la PNCP. 


\section{Recursos}

Para Knoepfel et al (2006), una política pública no se crea ni se lleva a cabo en el vacío. Los recursos de los que dispone y puede llegar a crear cada actor y todos ellos en su conjunto influyen desde el principio y de manera significativa en los resultados intermedios y finales de una política pública ${ }^{3}$.

Los autores plantean la existencia de diez recursos que a su juicio son específicos para el análisis de políticas públicas. Estos recursos son: fuerza (recurso violencia), derecho (recurso jurídico), personal (recurso humano), dinero (recurso económico), información (recurso cognitivo), organización (recurso interactivo), consenso (recurso confianza), tiempo (recurso cronológico), infraestructura (recurso patrimonio), apoyo político (recurso mayoría) ${ }^{3}$.

\section{Reglas Institucionales}

Para Knoepfel et al (2006), las instituciones o reglas institucionales son aquellas estructuras y reglas formales explícitas formalizadas jurídicamente y aquellas reglas informales implícitas, compartidas por los miembros de una organización, que establecen estructuras y procedimientos que facilitan o limitan la participación política de los individuos y los grupos ${ }^{3}$.

\section{Ciclo de la política pública y sus productos}

Knoepfel et al, en línea con la extensa bibliografía que acepta la existencia de un ciclo de vida de las políticas públicas, adapta dicho concepto considerando en su análisis que la política pública tiene un ciclo que comprende cuatro etapas y seis productos ${ }^{3}$. De manera adicional en su trabajo, contempla que la política pública es una variable dependiente de la interacción de tres variables independientes como son los actores, los recursos y las reglas institucionales, definidos anteriormente.

A continuación, se presentan las etapas y los productos que los autores consideran constituyen el ciclo de las políticas públicas.

Etapa 1: Inclusión en la agenda política. En esta etapa se hace la inclusión del problema público a resolver en la agenda gubernamental. Contiene un solo producto, la definición política del problema público.

- Producto 1. Definición política del problema público: Comprende la firme decisión de emprender una intervención pública y el marco desde el que se parte, es decir, la delimitación del "perímetro" del problema público y de las causas probables de este.

Etapa 2: Decisión-programación de las políticas públicas. En la etapa 2 la programación legislativa y reglamentaria de la intervención pública (con las decisiones pertinentes) se hace notoria. Esta etapa contiene dos productos:

- Producto 2. Programa de actuación político administrativo. Incluye las decisiones legislativas y reglamentarias tanto del Gobierno y la administración central como de los demás Gobiernos y administraciones públicas.

- Producto 3. Acuerdo de actuación político administrativo. Fija las competencias, las responsabilidades y los principales recursos de los actores públicos para la implementación del programa político-administrativo. 
Etapa 3: Implementación. Una etapa relevante es la de implementación del programa político-administrativo a través de planes de acción y de los actos formales que resulten de ello. En esta etapa existen dos productos.

- Producto 4. Planes de acción. Los cuales establecen las prioridades de implementación en el espacio geográfico y social, así como el tiempo.

- Producto 5. Actos de implementación. Que incluyen todas las actividades y las decisiones administrativas de aplicación.

Etapa 4: Evaluación de las políticas públicas y sus efectos. Evaluación de los efectos generados (impactos). Contiene un solo producto, los enunciados evaluativos.

- Producto 6. Enunciados evaluativos. Evidencian los eventuales cambios de comportamiento de los grupos-objetivo (impactos) y los efectos inducidos en los beneficiarios finales (outcomes) y permite valorar la pertinencia, la eficacia y la eficiencia de la política pública aplicada.

Teniendo en cuenta la metodología descrita, a continuación, se presentan los resultados de la aplicación de la misma en la PNCP: se describen los actores involucrados a través del diseño del llamado "triángulo de actores", describiendo las hipótesis de intervención y causal, se describen los recursos y las reglas institucionales de la PNCP y se describen los productos, 1, 2, 3 y 4 del Ciclo de la Política Pública.

\section{Resultados del análisis de la Política Nacional de Compe- titividad y Productividad 2008-2014 con la metodología de Knoepfel et al 2006}

\section{Descripción general de la PNCP}

Hacia inicios de la década del 2000, Colombia se ubicaba dentro de los indicadores mundiales de competitividad en una posición rezagada frente a otras naciones latinoamericanas, muy especialmente en el indicador del Foro Económico Mundial (FEM) según el cual, existe una relación alta y positiva entre competitividad y prosperidad social.

De acuerdo con dicho indicador, el país no solo era poco competitivo frente a otras naciones del continente, sino que también tenía una economía menos próspera, lo cual era un reflejo claro de la existencia de problemas sociales tales como la pobreza, la desigualdad o la inequidad entre muchos otros.

La PNCP parte de la idea de que, si se aumenta la competitividad, se logra aumentar la oferta de empleo formal. Si se aumenta el empleo formal, se logrará aumentar los ingresos reales de los colombianos que estén empleados, todo ello gracias al aumento de la productividad de las empresas. De esta forma, se aporta a la disminución de los niveles de pobreza nacional. En otras palabras, el problema que busca resolver la PNCP, es aportar a la disminución de los niveles de pobreza en el país, vía empleo formal generado por sectores económicos productivos.

Así mismo en la PNCP la competitividad, cuya forma menos controversial de medición es la productividad, se entiende como un complemento a las condiciones de un entorno proclive para el crecimiento económico, las cuales tienen que ver con seguridad física, seguridad jurídica y estabilidad macroeconómica ${ }^{11}$. 
En esos términos, el concepto de competitividad se aproxima a la definición planteada por el Foro Económico Mundial, en cuanto a que la competitividad es el conjunto de factores, instituciones y políticas públicas que determinan los niveles de productividad de un país ${ }^{22}$.

La PNCP, propone que la vía más relevante de aumento de la competitividad nacional es el denominado "proceso de transformación productiva", acogiendo lo dicho por Haussman y Klinger (2008) quienes analizaron las oportunidades de crecimiento nacional a partir de su participación en el mercado internacional, concluyendo que la productividad (producir más) y el mejoramiento de la calidad (producir mejor) se habían agotado como fuentes de crecimiento de la canasta exportadora colombiana, restando solamente la transformación productiva (producir nuevos productos) como única vía de aumento del valor de la producción del país dentro del mercado internacional².

Lo anterior se afirma a pesar que el estudio fue realizado a partir de datos de la canasta exportadora nacional, la cual para el año 2007 estaba dominada por los automotores, el carbón y el petróleo que representaban el 39,7\% del total de productos exportados ${ }^{1}$.

\section{Actores, recursos y reglas institucionales de la PNCP}

La figura 1, basada en lo que Knoepfel et al (2006) denomina el "triángulo de base" de una política pública, representa esquemáticamente el juego de actores de la PNCP.

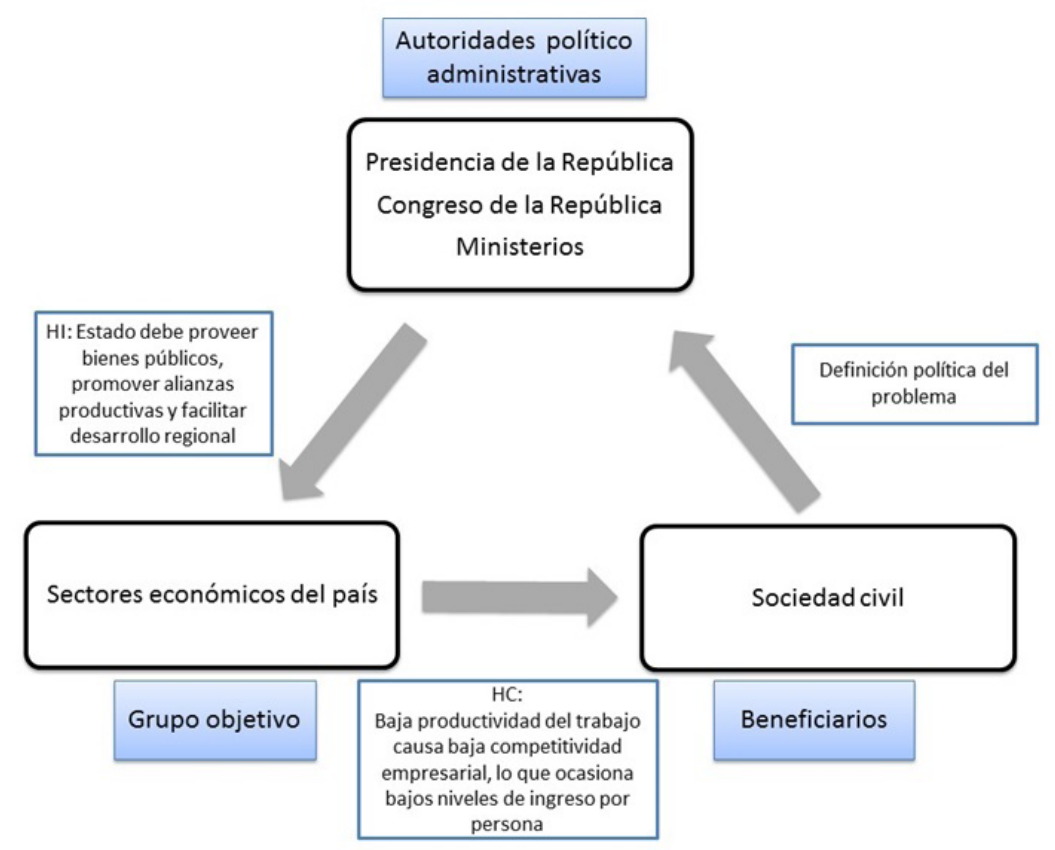

Figura 1. Triángulo de base de los actores involucrados en la PNCP. Elaboración propia, basado en Knoepfel (2006). HI: Hipótesis de intervención. HC: Hipótesis causal.

En la parte superior del gráfico se ubican lo que Knoepfel llama autoridades político administrativas, los actores públicos. Allí se encuentran la Presidencia de la República, el Congreso de la República y los diferentes ministerios. 
Como grupos objetivo (actores privados) se encuentran los distintos sectores económicos del país que son sobre los que en últimas deben ser más productivos y más competitivos. Como beneficiarios finales de acuerdo con la misma definición del problema colectivo en la PNCP, se ubica a la sociedad civil en su conjunto. Recordemos que esta es una política horizontal.

Dos elementos conceptuales propuestos por Knoepfel et al que permiten identificar mejor los nexos que existen entre los diferentes actores y la manera en que dichas relaciones pueden modificarse tras una intervención pública son las hipótesis causales o de causalidad y de intervención ${ }^{3}$.

Según Knoepfel, la hipótesis causal aporta una respuesta política a la cuestión de saber quién o qué es "culpable" u "objetivamente responsable" (es decir, dejando al margen posibles "culpabilidades" subjetivas) del problema colectivo a resolver. Al establecer una hipótesis causal se designan de hecho los grupos objetivos y los beneficiarios finales de la política pública ${ }^{3}$.

La hipótesis de intervención, trata de establecer cómo podría resolverse o atenuarse el problema colectivo a través de una política pública determinada, definiendo las modalidades de intervención estatal que influirán en las decisiones y las acciones de los grupos objetivo designados, de manera que vayan siendo compatibles con los objetivos políticamente planteados ${ }^{3}$.

Para el caso concreto, la hipótesis de intervención de la PNCP está formulada en el documento Conpes 3527 de 2008 de la siguiente manera: "siendo el sector privado el principal responsable de la productividad, el papel del Estado es facilitar los esfuerzos productivos del sector privado. Esta facilitación se puede dar en por lo menos tres niveles: 1) la provisión de bienes públicos que juegan el papel de insumos de producción para mejorar la productividad y competitividad de las firmas colombianas, 2) la promoción de alianzas productivas público-privadas, y 3) el fomento de la dimensión regional de la competitividad"11.

Para el caso de la hipótesis causal, el Informe Nacional de Competitividad del año 2007, postula que la muy baja productividad del trabajo en el país, es uno de los síntomas más relevantes de la baja competitividad ${ }^{1}$. Recordemos que de acuerdo con el documento Conpes 3527 de 2008, la productividad es la forma menos controversial de medir la competitividad ${ }^{11}$.

Según dicho Informe, la baja productividad del trabajo se debe a la insuficiente dotación de capital por trabajador, asociada a tasas de inversión relativamente bajas, además de la baja eficiencia con la que se usan los recursos disponibles El crecimiento de la productividad del trabajo, postula el Informe, es igual a la profundización del capital, la cual está directamente afectada por el crecimiento del stock de capital, más la acumulación de capital humano, más el crecimiento de la productividad factorial'".

De manera adicional, el Informe Nacional de Competitividad 2007 postula una serie de variables que afectan de manera directa el grado de eficiencia con la que se usan los recursos productivos en la economía. Entre las variables propuestas están los dias necesarios para empezar un negocio, el número de computadores por cada mil habitantes, el porcentaje de carreteras pavimentadas, los años de escolaridad en adultos y el número de impuestos por año, entre otros 1 . 
De acuerdo con lo anterior, la responsabilidad en los bajos niveles de productividad es compartida entre el Estado y el sector empresarial. En ese sentido, la PNCP debe estar orientada a modificar no solo el comportamiento del sector empresarial colombiano, sino también modificar en parte las acciones que el Estado en varios de sus niveles no solo de agregación político administrativa, sino de estructura funcional, hace en favor o en contra de ese sector.

Con respecto a los recursos principales de la PNCP estos son el Presupuesto Nacional de la República, a través de los presupuestos ministeriales, como recurso dinero y el Sistema Nacional de Competitividad e Innovación (SNCl) como recurso interactivo.

Por último y para el caso de la PNCP, las reglas institucionales formales principales son la Ley Nacional de Competitividad y Productividad 1253 de 2008 y el documento CONPES 3527 de 2008 que formula la PNCP.

Teniendo en cuenta el juego de poderes de los actores al momento de la formulación de la PNCP, es lógico pensar que esta estuviera mayormente destinada a sectores industriales del país y dirigida hacia los mercados internacionales.

\section{Producto 1 del ciclo de la Política Pública: Definición política del problema en la PNCP}

Uno de los problemas que la PNCP busca resolver, es el de la pobreza entendida como escasos recursos económicos, representado en bajos ingresos percápita, a través del aumento de la productividad de los sectores económicos nacionales.

Ahora bien, de acuerdo con mediciones de competitividad realizadas por agencias internacionales se identificaron doce ejes ${ }^{1}$ problemáticos que de acuerdo con el documento Conpes 3527 de 2008, impiden que el país sea más competitivo ${ }^{11}$. Estos ejes problemáticos se corresponden plenamente con los planes de acción que el documento Conpes contempla para la PNCP.

Sin embargo, en síntesis, lo que busca la PNCP es volver más competitivo al país. Lo anterior, según el Conpes 3527 de 2008, no solo permitirá al país mejorar en los escalafones de competitividad a nivel mundial, sino que también aportará en tres aspectos:

1. Aumentar la tasa de crecimiento con lo cual se lograría reducir la brecha existente con los países de ingresos medios altos,

2. Lograr insertar al país con éxito en la economía global, gracias al aumento de la competitividad, y

3. Generar oportunidades de empleo formal, combatiendo la pobreza y la desigualdad.

De esta forma, se considera que los aumentos de la competitividad son una herramienta para el desarrollo socioeconómico y la prosperidad colectiva del país ${ }^{11}$.

1. De acuerdo con el documento Conpes 3527/2008 existen doce ejes problemáticos, evidenciados por los resultados para el país de las mediciones de competitividad realizadas y reportadas en el mismo documento. Dichos ejes son: 1) poca sofisticación y baja agregación de valor en los procesos productivos; 2) baja productividad y capacidad de generación de empleo en los sectores formales; 3) baja productividad del sector agropecuario; 4) altos niveles de informalidad empresarial y laboral; 4) altos niveles de informalidad empresarial y laboral; 5) bajos niveles de innovación y absorción de tecnologías; 6 ) poca profundidad y sofisticación del mercado financiero: 7) deficiencias en la infraestructura de transporte y energía 8) baja calidad y poca pertinencia de la educación; 9) estructura tributaria poco amigable a la competitividad; 10) rezago en penetración de tecnologías de información y en conectividad; 11) degradación ambiental como limitante de la competitividad; 12) debilidad de la institucionalidad relacionada con la competitividad. 
En este punto, es válido aclarar que la definición actual de competitividad es resultado de la progresiva incorporación de dicho concepto en el discurso gubernamental, desde los tiempos de la Política de Apertura Económica.

Como resultado del proteccionismo de las décadas de 1980 y 1970, el mercado nacional se había saturado con productos locales, de tal manera que el poder de compra era inferior a la oferta. En adición, los precios de los productos nacionales habían incrementado con el tiempo, y el control de calidad se había deteriorado por falta de competencia. Frente a esta situación, la administración del presidente César Gaviria adoptó la Política de Apertura?.

El Gobierno Gaviria, modificó el sistema de importaciones, desmontando las restricciones administrativas y reduciendo el nivel general de aranceles. A partir de inicios de la década de 1990, se acelera el proceso de apertura económica, donde el 97\% de las posiciones arancelarias permitió la libre importación, provocando una invasión del mercado nacional con bienes importados que debido a sus bajos costos de producción ejercían presión sobre la producción nacional afectando los diferentes sectores económicos ${ }^{6}$.

La nueva ola de competencia generada por la introducción de productos extranjeros al mercado colombiano transformó por completo la relación de producción y demanda que había regido al país por varias décadas. Como resultado, muchas industrias no lograron sobrevivir a la competencia, y sectores enteros de producción desaparecieron?.

En el Gobierno Samper (1994-1998), se inicia formalmente el proceso de la incorporación de la competitividad como elemento significativo en la economía nacional, mediante la creación de la Comisión Nacional de Competitividad y los Acuerdos Sectoriales de Competitividad. En el Gobierno Pastrana (1998-2002), se llega a formular la Política Nacional de Productividad y Competitividad, pero solo es ante la necesidad manifiesta de asumir retos y planes de acción concretos frente a la firma del Tratado de Libre Comercio con Estados Unidos que el Gobierno Uribe (2002-2006) crea el llamado Sistema Nacional de Competitividad, creando una serie de organismos e instituciones que regulan, conceptualizan y operativizan la competitividad ${ }^{12}$.

Dicho sistema, llamado Sistema Nacional de Competitividad, Ciencia, Tecnología e Innovación SNCCTI, luego de la fusión con el Sistema de Ciencia, Tecnología e Innovación, a través del Artículo 186 de la Ley 1753 de 2015 "por la cual se expide el Plan Nacional de Desarrollo 2014-2018 Todos por un nuevo país"10, fue el encargado de formular la PNCP a través de la Comisión Nacional de Competitividad e Innovación ${ }^{12}$.

El eje fundamental del SNCCTI es la Comisión Nacional de Competitividad, Ciencia, Tecnología e Innovación, el cual es el órgano asesor del Gobierno Nacional y de concertación entre este, las entidades territoriales y la sociedad civil en temas relacionados con la productividad y competitividad del país y de sus regiones, con el fin de promover el desarrollo económico ${ }^{19}$. 


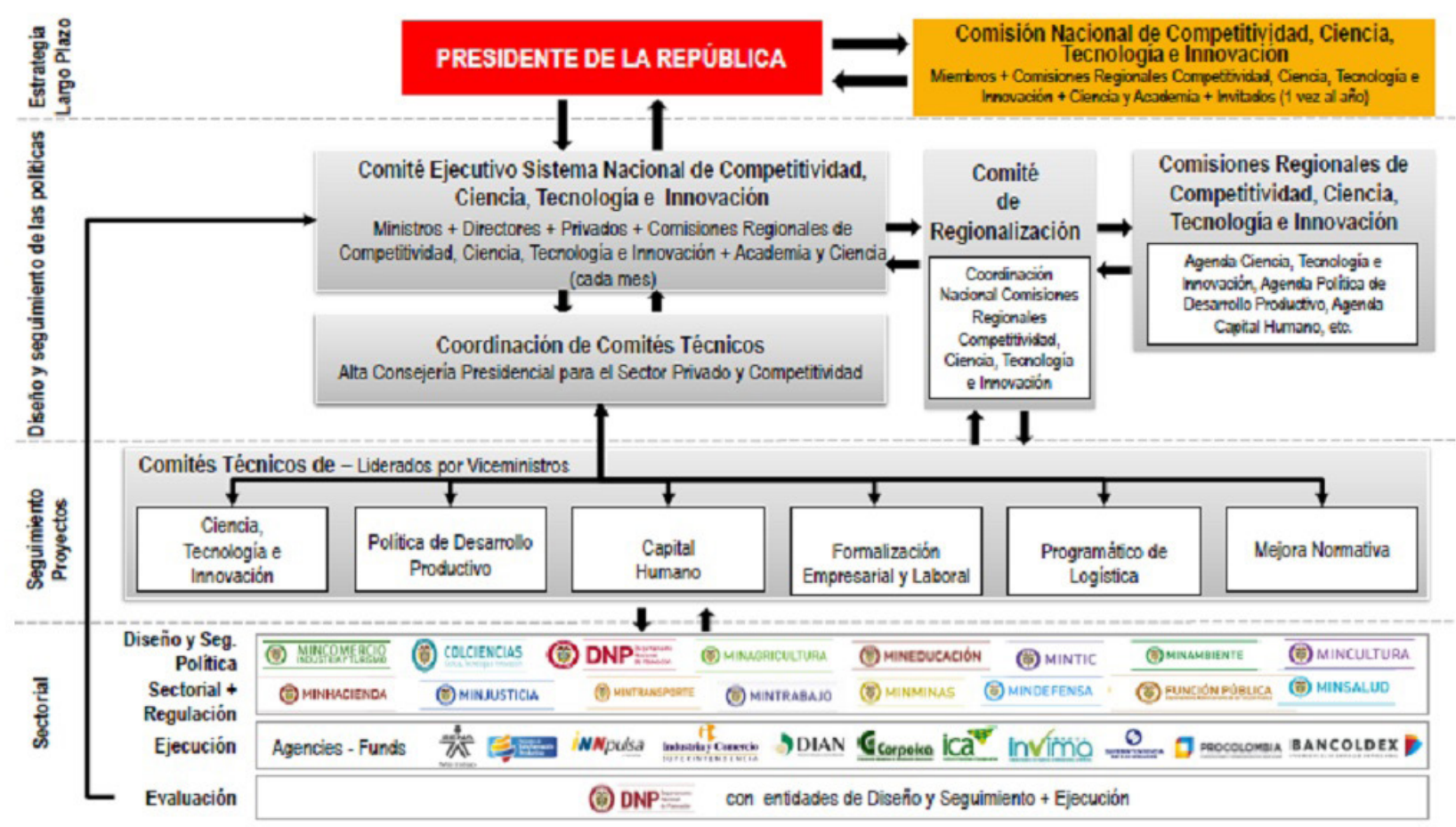

Figura 2. Gobernanza del SNCCTI. Fuente: República de Colombia Presidencia de la República (2016).

Una de las acciones de dicha Comisión ha sido crear lineamientos generales para la PNCP, entre los cuales se halla la visión de competitividad del país: "En 2032 Colombia será uno de los tres países más competitivos de América Latina y tendrá un elevado nivel de ingreso por persona, equivalente al de un país de ingresos medios altos, a través de una economía exportadora de bienes y servicios de alto valor agregado e innovación, con un ambiente de negocios que incentive la inversión local y extranjera, propicie la convergencia regional, mejore las oportunidades de empleo formal, eleve la calidad de vida y reduzca sustancialmente los niveles de pobreza"11.

Analizando la visión de competitividad del país, en la cual se basa de manera fundamental la esencia de la PNCP, es válido preguntarse acerca de los indicadores de pobreza en Colombia, considerando que disminuir los mismos es uno de los aportes directos a los que le apunta dicha Política.

Las medidas monetarias de la pobreza se basan en la determinación de un nivel de ingreso que garantice un estandar de vida minimo en el país ${ }^{15}$. La figura 3 resume los porcentajes de pobreza monetaria para el país en el periodo 1991-2014. Revisando dichos indicadores, no era descabellado pensar que la pobreza fuera uno de los temas principales a los cuales los aumentos en los niveles de competitividad debería ayudar a resolver.

La serie no presenta los datos para los años 2006 y 2007, ya que para el año 2006, se inició el cambio de la herramienta (se pasó de la Encuesta Continua de Hogares -ECH- a la Gran Encuesta Integrada de Hogares -GElH-) que se aplica para la toma de datos de medición de pobreza monetaria. Puntualmente para el año 2006, no había datos suficientes para el análisis y para el año 2007, el nuevo instrumento aún requería ajustes ${ }^{13}$. 
Para el año 2008, (año en que se formula la PNCP), el porcentaje de la población colombiana catalogada como pobre monetariamente, era de $42 \%$. En términos absolutos y teniendo en cuenta que la población colombiana total proyectada por el Departamento Administrativo Nacional de Estadística (DANE) para el año 2008 era de 44.451.147, para ese año, habían aproximadamente 18.670.000 colombianos pobres.

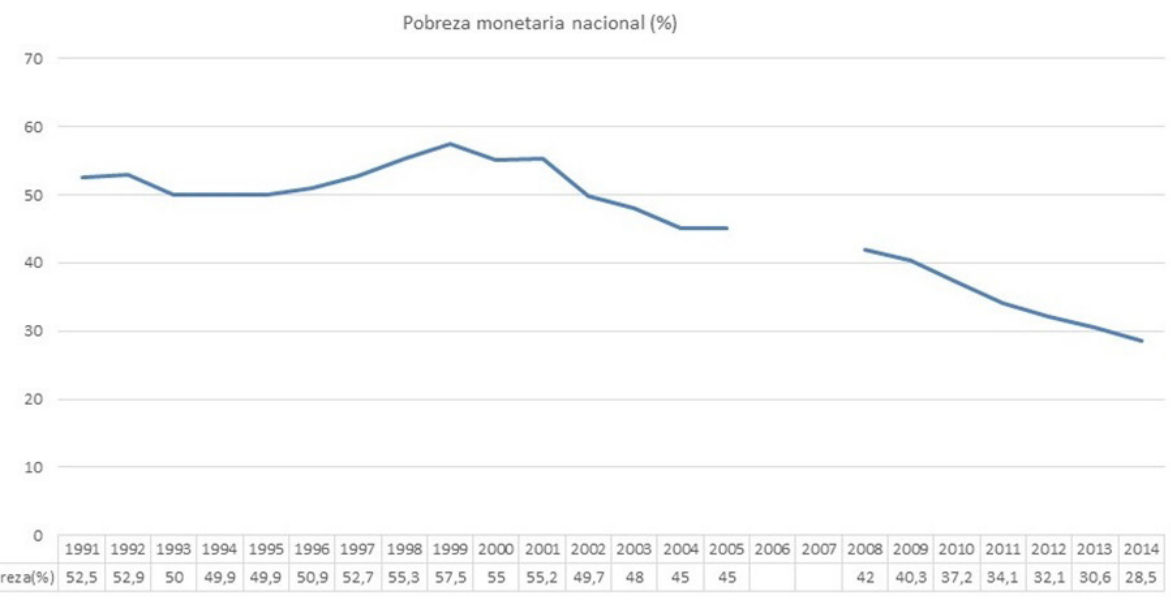

Figura 3. Niveles de pobreza monetaria en Colombia (1991-2014). Elaboración propia a partir de Departamento Nacional de Planeación (2007) y Departamento Administrativo Nacional de Estadistica (2016).

La tendencia está dirigida a una disminución en el porcentaje de población pobre monetariamente en el país, pero ello no se puede decir expeditamente que ha sido gracias a la acción de la PNCP, sino que deben analizarse cada una de las variables que inciden sobre los niveles de pobreza monetaria. En ese sentido, no se puede afirmar que la PNCP ha aportado sustancialmente a la disminución de pobreza monetaria, pero si se puede afirmar que muy posiblemente haya sido uno de los motores que han aportado a la disminución de la misma, a través del fomento a los sectores económicos para que aumenten sus niveles de productividad.

\section{Productos 2 y 3 del ciclo de la Política Pública: Programación político administrativa y acuerdo político administrativo en la PNCP}

La Programación Político Administrativa (PPA) si bien quedó plasmada en el CONPES 3527 de junio de 2008 y en la Ley 1253 del 27 de noviembre de 2008- Ley Nacional de Competitividad y Productividad, se le ha sumado durante los últimos años las leyes 1450 de 2011 y 1753 de 2015, leyes del Plan Nacional de Desarrollo.

De acuerdo con la Ley 1253 de 2008, es obligación del Gobierno Nacional, velar por la formulación y ejecución de políticas y programas que tengan impacto en la productividad y la competitividad y ordena que tanto en el Plan Nacional de Desarrollo, como en los Planes Territoriales de Desarrollo existan mecanismos que redunden en el aumento de la productividad y la competitividad ${ }^{8}$.

Además, señala también que es obligación al inicio y al final de cada administración nacional presentar un informe sobre la competitividad internacional del país en los factores transversales, regionales y sectoriales ${ }^{8}$. 
Así mismo, en las leyes del Plan Nacional de Desarrollo tambien han quedado planteado el juego de actores de los Acuerdos Políticos Administrativos (APA), ya que si bien los Planes Nacionales y Territoriales de Desarrollo se generan primordiamente a partir de las ideas de los candidatos elegidos, el juego en cuanto a competitividad, según dichas leyes ha quedado en manos de las Comisiones Regionales de Competitividad (CRC), con lo que dichas leyes y los decretos que las desarrollan, se constituyen en el APA de la PNCP.

De acuerdo con el Decreto 1500 del 13 de julio de 2012, decreto que desarrolla lo que el articulo 33 de la Ley 1450 de 2011, "por la cual se expide el Plan Nacional de Desarrollo 2010-2014", estableció a nivel legal en cuanto a las competencias de las CRC y la coordinación y articulación al interior de cada departamento, los CRC son organos que coordinan y articulan al interior de los departamentos a los principales actores de los sectores públicos y privados en temas de competitividad, productividad e innovación y tienen a su cargo la formulación de los Planes Regionales de Competitividad (PRC) ${ }^{18}$.

Por otro lado, los artículos noveno y decimosegundo del mismo Decreto, postulan que las CRC coordinan y articulan la implementación de las políticas de desarrollo productivo, de competitividad y productividad al interior de los departamentos y que los Planes Territoriales de Desarrollo deben recoger las propuestas que salgan de los PRC?.

Finalmente, en el artículo 186 de la Ley 1753 de 2015, "por la cual se expide el Plan Nacional de Desarrollo 2014-2018", se introdujo un cambio en el juego de actores en la denominada gobernanza del Sistema, redefiniendose toda la composición de la misma luego de la fusión del Sistema Nacional de Competitividad e Innovación con el Sistema de Ciencia, Tecnología e Innovación ${ }^{10}$, la cual fue expuesta anteriormente.

Así, a partir de la entrada en vigencia de dicha Ley, las distintas instancias departamentales que promueven agendas de competitividad, productividad, ciencia, tecnología e innovación, tales como los Consejos Departamentales de Ciencia, Tecnología e Innovación (Codecti), Comités Universidad-Empresa-Estado, Comités de Biodiversidad, Redes Regionales de Emprendimiento, Consejos Regionales de PYME, Consejos Ambientales Regionales, Comités de Seguimiento a los Convenios de Competitividad y las demás que sean promovidas por el Gobierno Nacional se integraron a las Comisiones Regionales de Competitividad en cada departamento, con el propósito de articular sus agendas de trabajo ${ }^{10}$.

Ahora, surge la duda acerca de si dentro de esos escenarios de decisión político administrativo como son los CRC, tengan cabida organizaciones de productores pecuarios, más especificamente organizaciones de productores apícolas o en su defecto si en ellas tienen asiento permanente las secretarias técnicas de los Comités Regionales de la Cadena Productiva de las Abejas y la Apicultura (CPAA). 


\section{Producto 4 del ciclo de la Política Pública: Planes de acción de la PNCP}

En los quince planes de acción ${ }^{2}$ contemplados en la PNCP, que en gran medida corresponden a los doce eje problemáticos que impiden que Colombia sea más competitiva y que ya fueron enunciados en la definición Política del problema público, uno de ellos hace referencia directa al sector agropecuario.

El plan "competitividad en el sector agropecuario", diseñado por el Ministerio de Agricultura y Desarrollo Rural basandose en los objetivos generales de la PNCP, matizandolos para el sector, propone cuatro ejes estrategicos y una serie de objetivos por eje que se desarrollan en matrices de actividades y productos que en la mayoría de los casos incluye indicadores de seguimiento, metas, plazos y responsables.

A continuación, se presentan los cuatro ejes y los objetivos específicos por eje:

\section{Mejorar la productividad y eficiencia en los sistemas de producción agropecuaria:}

a. Mejorar la innovación tecnológica en los sistemas de producción agropecuarios.

b. Ampliar la cobertura y mejorar la operación de los sistemas de riego y drenaje.

c. Mejorar la eficiencia en el uso del suelo.

d. Aumentar la eficiencia de las actividades de comercialización y logística de las cadenas agropecuarias.

e. Reducir los costos de producción de los sistemas productivos agropecuarios.

\section{Mejorar el acceso real de la producción agropecuaria a los mercados interna- cionales:}

a. Mejorar estatus sanitario de la producción agropecuaria.

b. Abrir los mercados para la producción agropecuaria y asegurar su permanencia.

\section{Propender por la estabilidad del ingreso de los productores y el incremento de las inversiones en el campo:}

a. Estabilidad en los ingresos de los productores.

b. Incrementar el financiamiento.

c. Mejorar el desempeño de los mercados

\section{Sectores de clase mundial en el sector agropecuario ${ }^{10}$}

Del total de matrices de productos y actividades contemplados en el plan de acción del sector agropecuario en la PNCP, las tablas 2, 3 y 4, presentan algunos apartes de dicha matriz que representan una posibilidad u oportunidad para el sector apícola colombiano. 
Tabla 2. Matriz de productos y actividades del plan de acción para el sector agropecuario en la PNCP, Eje estratégico No. 1.

\section{Eje 1. Mejorar la productividad y eficiencia en los sistemas de producción agropecuaria}

Producto

Actividad

Elaboración de agendas de innovación tecnológica con los actores de la cadena identificando limitantes tecnológicos

Agendas de innovación tecnológica por cadena productiva

\section{Convocatorias de ciencia y tecnología}

Financiación de proyectos de innovación tecnológica a través de convocatorias

Fomento a la producción y suministro de los productos obtenidos en las Agendas de Innovación

Acuerdos de aprovechamiento e innovación por cadena productiva
Indicador, meta, tiempo y entidad responsable

\begin{tabular}{|c|c|c|}
\hline & & Número de beneficiarios, MADR. \\
\hline $\begin{array}{l}\text { Fomento al uso de la } \\
\text { asistencia técnica en la } \\
\text { actividad agropecuaria }\end{array}$ & $\begin{array}{l}\text { Incentivo a la Asistencia } \\
\text { Técnica de Agro Ingreso } \\
\text { Seguro }\end{array}$ & $\begin{array}{l}\text { Número de departamentos con } \\
\text { empresas habilitadas por el MADR } \\
\text { para prestar el servicio / Número } \\
\text { departamentos Total, } 100 \%, 2010 \text {, } \\
\text { MADR. }\end{array}$ \\
\hline $\begin{array}{l}\text { Desarrollo del ICR hacia } \\
\text { líneas de crédito de } \\
\text { Innovación tecnológicas }\end{array}$ & $\begin{array}{l}\text { Implementación de las líneas } \\
\text { de ICR para innovación } \\
\text { tecnológica }\end{array}$ & $\begin{array}{l}\text { Porcentaje de ICR para las líneas } \\
\text { de innovación, MADR. }\end{array}$ \\
\hline $\begin{array}{l}\text { Fortalecimiento de } \\
\text { la infraestructura de } \\
\text { producción de cosecha y } \\
\text { poscosecha }\end{array}$ & $\begin{array}{l}\text { Implementación de líneas } \\
\text { de crédito para compra de } \\
\text { maquinaria e infraestructura }\end{array}$ & $\begin{array}{l}\text { Valor de los Créditos (millones } \\
\text { de pesos) línea para compra de } \\
\text { maquinaria y construcción de } \\
\text { infraestructura, MADR }\end{array}$ \\
\hline \multirow[t]{2}{*}{$\begin{array}{l}\text { Fortalecimiento de la } \\
\text { Educación Superior Rural }\end{array}$} & \multirow{2}{*}{$\begin{array}{l}\text { 1. Creación de nuevos cupos } \\
\text { en educación superior a } \\
\text { través de los Ceres } \\
\text { 2. Ampliar la cobertura del } \\
\text { programa Ceres a través de } \\
\text { su área de influencia. } \\
\text { 3. Ampliar la participación } \\
\text { de programas técnicos } \\
\text { profesionales y tecnológicos } \\
\text { en los Ceres }\end{array}$} & $\begin{array}{l}50.000 \text { cupos a } 2010 \text { MEN, } 18300 \\
\text { cupos se han generado a julio de } \\
\text { 2008; } 500 \text { municipios cubiertos por } \\
\text { el programa Ceres a través de su } \\
\text { área de influencia a } 2010 \text {, MEN, a } \\
\text { julio de } 2008 \text { se han cubierto } 480 \\
\text { municipios. }\end{array}$ \\
\hline & & $\begin{array}{l}65 \% \text { de los programas ofrecidos en } \\
\text { los Ceres son Ty T, a 2010, MEN, a } \\
\text { julio de } 2008 \text { la participación de los } \\
\text { programas Ty T en los Ceres es de } \\
\text { un } 47 \% \text {. }\end{array}$ \\
\hline
\end{tabular}


Tabla 3. Matriz de productos y actividades del plan de acción para el sector agropecuario en la PNCP. Eje estratégico No. 2.

Eje 2. Mejorar el acceso real dela producción agropecuaria a los mercados internacionales

\begin{tabular}{lll}
\hline \multicolumn{1}{c}{ Producto } & \multicolumn{1}{c}{ Actividad } & $\begin{array}{c}\text { Indicador, meta, tiempo, } \\
\text { entidad responsable }\end{array}$ \\
\hline $\begin{array}{l}\text { Establecimiento de sistemas } \\
\text { de trazabilidad para productos } \\
\text { agropecuarios. }\end{array}$ & $\begin{array}{l}\text { Diseño e implementación de } \\
\text { los Sistemas }\end{array}$ & $\begin{array}{l}\text { Sistemas de trazabilidad } \\
\text { implementados. ICA, 2019. }\end{array}$ \\
\hline $\begin{array}{l}\text { Zonas de baja prevalencia de } \\
\text { plagas. }\end{array}$ & Vigilancia y control del ICA & Zonas, 7, año 2010, ICA \\
\hline $\begin{array}{l}\text { Actualización sistema de registro } \\
\text { de pesticidas y la determinación } \\
\text { de límites máximos de residuos. }\end{array}$ & $\begin{array}{l}\text { Implementación del Sistema } \\
\text { De Registro }\end{array}$ & $\begin{array}{l}\text { Sistema de registro } \\
\text { actualizado, ICA. }\end{array}$ \\
\hline $\begin{array}{l}\text { Mejoramiento la capacidad de } \\
\text { los sistemas de diagnóstico } \\
\text { nacionales. }\end{array}$ & $\begin{array}{l}\text { 1. Acreditar los laboratorios } \\
\text { nacionales de referencia. } \\
\text { 2.Conformar las redes } \\
\text { nacionales y categorizar los } \\
\text { laboratorios }\end{array}$ & $\begin{array}{l}\text { Número de laboratorios } \\
\text { nacionales de referencia } \\
\text { acreditados y vinculados a } \\
\text { redes internacionales. }\end{array}$ \\
\hline
\end{tabular}

Fuente: elaboración propia a partir de CONPES 3527 de 2008

Tabla 4. Matriz de productos y actividades del plan de acción para el sector agropecuario en la PNCP. Eje estratégico No. 3.

Eje 3. Propender por la estabilidad del ingreso de los productores y el incremento de las inversiones en el campo

\begin{tabular}{|c|c|c|}
\hline Producto & Actividad & $\begin{array}{c}\text { Indicador, meta, tiempo, } \\
\text { entidad responsable }\end{array}$ \\
\hline $\begin{array}{l}\text { Fortalecimiento del seguro } \\
\text { agropecuario. }\end{array}$ & $\begin{array}{l}\text { Implementación del seguro } \\
\text { comercial }\end{array}$ & $\begin{array}{l}\text { Área (has) total cubierta por } \\
\text { el seguro. } \\
\text { Número de productos } \\
\text { funcionando con seguro. }\end{array}$ \\
\hline $\begin{array}{l}\text { Incremento del valor de los } \\
\text { créditos desembolsados para el } \\
\text { sector agropecuario }\end{array}$ & $\begin{array}{l}\text { Fomento de las colocaciones } \\
\text { de crédito de redescuento para } \\
\text { el sector a través de Finagro }\end{array}$ & $\begin{array}{l}\text { Valor de los créditos para el } \\
\text { sector a través de Finagro. }\end{array}$ \\
\hline $\begin{array}{l}\text { Acuerdos regionales de } \\
\text { competitividad para cadenas } \\
\text { productiva. }\end{array}$ & $\begin{array}{l}\text { 1. Hacer seguimiento a los } \\
\text { acuerdos firmados. } \\
\text { 2. Convocar permanentemente } \\
\text { a los miembros de las cadenas } \\
\text { productivas en las regiones. }\end{array}$ & $\begin{array}{l}\text { Número de acuerdos de } \\
\text { competitividad. }\end{array}$ \\
\hline $\begin{array}{l}\text { Sistema de información } \\
\text { de la oferta agropecuaria } \\
\text { consolidado }\end{array}$ & $\begin{array}{l}\text { Coordinación entre MADR- } \\
\text { DANE y CCI para fortalecer } \\
\text { y consolidar la Encuesta } \\
\text { Nacional Agropecuaria. ENA }\end{array}$ & $\begin{array}{l}\text { Sistema operando, MADR y } \\
\text { DANE. }\end{array}$ \\
\hline $\begin{array}{l}\text { Red información estratégica } \\
\text { del sector agropecuario } \\
\text { consolidada Agronet. }\end{array}$ & $\begin{array}{l}\text { 1. Implementar módulo de } \\
\text { pequeños productores. } \\
\text { 2. Ventana de agronegocios }\end{array}$ & $\begin{array}{l}\text { Sistema operando, MADR y } \\
\text { DANE. }\end{array}$ \\
\hline $\begin{array}{l}\text { Sistema de información de } \\
\text { precios. SIPSA }\end{array}$ & Mejoramiento continuo & $\begin{array}{l}\text { Sistema operando y con } \\
\text { nuevos instrumentos } \\
\text { virtuales, MADR, } 2010 .\end{array}$ \\
\hline
\end{tabular}

Fuente: elaboración propia a partir de Conpes 3527 de 2008 
Como se pudo observar en la matriz de productos y actividades, la PNCP confirma lo dicho por Martínez Ortíz (2011) frente a las políticas horizontales, quien las concibe como las herramientas que tiene el Estado para proveer un conjunto de bienes públicos que mejoren la productividad a todos los actores privados ${ }^{5}$.

En ese sentido la PNCP presenta para el caso concreto, una serie de herramientas transversales para todos los sectores agrícolas, pecuarios, pesqueros y forestales. De esa forma, el sector apícola en Colombia, si bien no está del todo explicitado en el plan de acción del sector agropecuario en la Política Nacional de Competitividad y Productividad, si tiene dentro de la misma, oportunidades que deben ser aprovechadas.

Esta situación de transversalidad se evidencia al momento de revisar el seguimiento a los planes de acción de la PNCP reportado en el documento Conpes 3686 de 2010 el cual, dando cumplimiento a la obligatoriedad de rendición de cuentas planteada por la Ley 1253 de 2008, hace un balance en 13 de los 15 planes de acción aportando adicionalmente, algunos ajustes encaminados al fortalecimiento de la ejecución de la política y que para el apartado del sector reporta lo grueso de las inversiones y acciones realizadas, pero no especifica por subsector ${ }^{12}$.

Los avances presentados en cada uno de los ejes del plan de acción para el sector agropecuario diseñado en 2008, son:

\section{Mejorar la productividad y la eficiencia en los sistemas de producción y comercia- lización agropecuaria:}

El plan originalmente contemplaba el trabajo en cinco frentes de acción, sin embargo, entre el 2008 y el 2010 se trabajaron en seis áreas:

a. Promoción de la innovación tecnológica y b) Fortalecimiento de los encadenamientos productivos.

Durante el periodo se generaron 17 Agendas Prospectivas de Investigación en Cadenas Productivas Agroindustriales y se financiación de 591 proyectos de investigación en 27 cadenas productivas, sin mencionar en detalle cuales.

b. Ampliación de la cobertura de la operación de sistemas de riego.

Ejecución del programa Agro Ingreso Seguro (AIS), establecido por la ley 1133 de 2007, el cual tiene dos componentes: Apoyos Económicos Directos y Apoyos para la Competitividad.

Dentro del componente de Apoyos para la Competitividad se establecieron los siguientes instrumentos: Apoyos vía crédito (Línea Especial de Crédito LEC), Incentivo de Capitalización Rural (ICR), Riego y Asistencia Técnica. Para el desarrollo de este objetivo, se puso en marcha las convocatorias para cofinanciación de proyectos con destino a la ampliación y mejoramiento operativo de sistemas de riego y drenaje y construcción de distritos de riego.

c. Mejoramiento en la eficiencia del uso del suelo:

En este punto se reportó la ampliación de la cobertura de área bajo riego, mediante la construcción y rehabilitación de distritos de riego y drenaje de mediana y gran escala, por parte del Instituto Colombiano de Desarrollo Rural (Incoder). 
d. Incremento de la inversión en infraestructura para la producción y la comercialización. El documento reporta hubo un proceso de otorgamiento de 3.162 créditos LEC a través del programa AIS y de 12.811 incentivos ICR, del mismo programa.

e. Reducción de costos de producción.

Entrega de créditos LEC para la compra de maquinaria e infraestructura para la producción y el establecimiento del Régimen de Libertad Vigilada de los precios de los insumos ${ }^{11}$.

\section{Mejorar el acceso real de la producción agropecuaria a los mercados interna- cionales:}

En el plan original se contemplaban tan solo dos áreas de trabajo, en el informe del Conpes 3668, se registraron avances en tres:

a. Mejorar estatus sanitario y fitosanitario del país. Gestión de 88 protocolos de exportación agrícola y pecuaria: 56 para exportación de cacao, uchuva, papaya entre otros con destino a trece países y 32 protocolos para exportación de bovinos, semen bovino, carne bovina, harina de pescado, entre otros. Adicional se obtuvo la certificación por parte de la Organización Mundial de Sanidad Animal (OIE) de país libre de Aftosa con vacunación.

b. Conseguir acreditaciones internacionales. Acreditación internacional de dos laboratorios colombianos de referencia (LANIA- Laboratorio Nacional de Insumos Agrícolas, LANIP- Laboratorio Nacional de Insumos Pecuarios) contribuyendo al mejoramiento del estatus sanitario de la producción agrícola.

c. Implementar sistemas de trazabilidad. Implementación de la primera fase del Sistema de Identificación e Información de Ganado Bovino (Sinigán) ${ }^{11}$.

\section{Propender por la estabilidad del ingreso de los productores y el incremento de las inversiones en el campo:}

En este punto se trabajó en tres áreas:

a. Estabilidad en los ingresos de los productores. Atención de 268.314 beneficiarios del programa AIS, de una cifra proyectada en 339.000 beneficiarios, y 1.376 talleres de acompañamiento municipal con el cual se pretendía fortalecer el conocimiento del acceso a los beneficios del programa.

b. Incrementar el financiamiento. Asignación de $\$ 1,7$ billones de pesos, por medio de la adjudicación de créditos LEC del programa AIS, adicionales a los $\$ 13,4$ billones de pesos en créditos de Finagro y el Banco Agrario, asignados entre el 2006 y mayo de 2010.

c. Mejorar el desempeño de los mercados. Consolidación del Sistema de Información Agropecuaria, fortaleciendo la publicación de la Encuesta Nacional Agropecuaria (ENA) y la página web Agronet ${ }^{12}$.

Sin lugar a dudas, los tres planes de acción expuestos en éste trabajo representan oportunidades especialmente para el eslabón primario de la Cadena Productiva de las Abejas. 
Las actividades del primer eje estratégico, están enfocadas para el fortalecimiento de la dotación de capital físico y humano, representados especialmente en subsidios a la producción: asistencia técnica, maquinaria, infraestructura, formación y capacitación y de acuerdo con lo reportado en el Conpes 3668 de 2010, existe el presupuesto asignado para la consecusion de las metas, sin embargo, se haría necesario identificar a plenitud si el mismo se está destinando también para el sector apícola.

Las actividades del segundo eje estratégico, tienden a asegurar la calidad de todos los eslabones de la Cadena: trazabilidad, vigilancia a plagas, acreditación de laboratorios, sin embargo, y de acuerdo con lo reportado en el Conpes 3668 de 2010, estas actividades se están direccionando a algunos sectores pecuarios (carne, leche), lo que en definitiva representaría una desventaja en la práctica para el sector apícola, aun cuando en lo formal la PNCP no tenga carácter sectorial.

Finalmente, las actividades del tercer eje estratégico, tienen un enfoque hacia el eslabón primario (seguro agropecuario) y otro hacia los demás eslabones (sistema de precios, información de la oferta agropecuaria), y como en el caso del segundo eje estrategico, los beneficios podrían estar siendo destinados a sectores pecuario priorizados en la agenda gubernamental.

La tarea de definir en detalle cuales de estas actividades han impactado a que cantidad de productores apícolas y en que departamentos, es una labor que corresponde a las organizaciones gremiales del sector a nivel nacional y territorial.

Sin embargo, como se observó en el informe de seguimiento de la PNCP de 2010 no queda lo suficientemente claro el impacto que estas actividades puedan tener en la Cadena Productiva de las Abejas y la Apicultura.

Con respecto a los enunciados evaluativos que de acuerdo con la metodología de Knoepfel et al corresponden al producto 6 del Ciclo de la Política Pública, este no es posible generar para el presente trabajo, dada la poca información pública disponible que se tiene al respecto de si los productos y las actividades planteadas por la PNCP han tenido impacto en los productores apícolas y cual ha sido la magnitud del mismo. Estos enunciados evaluativos, corresponden a las organizaciones gremiales apícolas.

\section{A modo de conclusión}

Como se evidenció en el presente trabajo, las políticas públicas son estrategias de acción estatal y social dinámicas, sujetas a la interacción de los actores que estén involucrados en la construcción de las mismas.

Metodologías como la de Knoepfel et al se convierten en aportes significativos para analizar las interacciones existentes entre ellos en aras de definir de qué manera está diseñada la política y de qué forma esta logra cumplir sus objetivos e impactar verdaderamente a la población objetivo.

Para el caso puntual de la relación entre la PNCP, que es un política horizontal que trata de ofrecer herramientas comunes a todos los sectores económicos del país, y el sector apícola colombiano, si bien se demostró que algunos de los planes de acción ofrecen verdaderas oportunidades para distintos eslabones de la cadena productiva, se hace necesario que las organizaciones gremiales sean capaces de interactuar con las autoridades político administrativas y ser parte de sus beneficios, considerando que los mismos se convierten en oportunidades que el entorno 
les ofrece a las empresas y los productores apícolas, oportunidades que pueden ser involucradas en los planes estratégicos de los mismos.

Sin embargo, previo al proceso de interacción con las autoridades, las organizaciones gremiales deben considerar refinar los diagnósticos sobre su actividad y sus planes estratégicos sectoriales de acción, no sólo para tener información de mejor calidad y más certera, sino también para identificar con exactitud las dimensiones de los problemas que presentan los distintos eslabones de la Cadena en los distintos territorios dónde se asienta la actividad apícola.

\section{Referencias}

1. Consejo Privado de Competitividad. (2007). Informe Nacional de Competitividad 2007. Bogotá: Consejo Privado de Competitividad.

2. Haussman, R., \& Klinger, B. (2008). Achieving export-led growth in Colombia. Harvard Universitty: Center for International Development.

3. Knoepfel, P., Larrue, C., Varone, F. Analyse et politage des politiques publiques. Zurich, Verlag Rüegger 2006.

4. Martínez Anzola, T. (2006). Diagnóstico de la actividad apícola y la crianza de abejas en Colombia. Bogotá: Ministerio de Agricultura y Desarrollo Rural-Instituto Interamericano de Cooperación para la Agricultura IICA.

5. Martínez Ortíz, A. (2011). Políticas sectoriales y horizontales en la estrategia de competitividad en Colombia (1994-2010). En A. Martínez Ortíz, \& J. A. Ocampo, Hacia una política industrial de nueva generación para Colombia (págs. 41-56). Bogotá: Coalición para la promoción de la industria colombiana.

6. Nájar, A. I. (2006). Apertura económica en Colombia y el sector externo (19902004). Apuntes del CENES, 77-98.

7. República de Colombia, Subgerencia Cultural del Banco de la República. (2015). Apertura Económica. Recuperado el 8 de diciembre de 2017, de Biblioteca Luis Ángel Arango del Banco de la República: http://www.banrepcultural.org/blaavirtual/ayudadetareas/poli/apertura-economicahtm

8. República de Colombia. Congreso de la República. (27 de Noviembre de 2008). Ley 1253 de 2008. Por el cual se regula la productividad y competitividad y se dictan otras dispocisiones. Bogotá D.C., Colombia.

9. República de Colombia. Congreso de la República. (2011). Ley 1450 de 2011. Por la cual se expide el Plan Nacional de Desarrollo 2010-2014. Bogotá D.C., Colombia.

10. República de Colombia. Congreso de la República. (2015). Ley 1753 de 2015. Por la cual se expide el Plan Nacional de Desarrollo 2014-2018. Bogotá, Colombia.

11. República de Colombia. Consejo Nacional de Politica Económica y Social. (2008). Documento CONPES 3527 Política Nacional de Competitividad y Productividad. 2008: Departamento Nacional de Planeación República de Colombia. 
12. República de Colombia. Consejo Nacional de Politica Económica y Social. (2010). Documento Conpes 3668 Informe de seguimiento a la Politica Nacional de Competitividad y productividad. Bogotá: Departamento Nacional de Planeación República de Colombia.

13. República de Colombia. Departamento Administrativo Nacional de Estadística. (Agosto de 2009). Misión para el empalme de las series de empleo, pobreza y desigualdad. Resultados fase 1: Emplame de la series de mercado laboral, pobreza y desigualdad. Recuperado el 22 de enero de 2018, de Departamento Administrativo Nacional de Estadistica: http://www.dane.gov.co/files/noticias/ Presentacion pobreza dane DNP.pdf

14. República de Colombia. Departamento Administrativo Nacional de Estadísticas. (2 de Marzo de 2016). Boletín técnico. Pobreza monetaria y multidimensional en Colombia 2015. Recuperado el 22 de marzo de 2018, de Departamento Administrativo Nacional de Estadística: https://www.dane.gov.co/files/investigaciones/ condiciones vida/pobreza/bol pobreza 15 .pdf

15. República de Colombia. Departamento Nacional de Planeación. (2007). Pobreza y Desigualdad en Colombia. Diagnóstico y estrategias. Bogotá.

16. República de Colombia. Ministerio de Agricultura y Desarrollo Rural. (20 de Febrero de 2008). Acta del Acuerdo Sectorial de Competitividad de la Cadena Productiva de la Apicultura y las Abejas. Bogotá, Colombia.

17. República de Colombia. Ministerio de Agricultura y Desarrollo rural. (Marzo de 2011). Plan Estrategico de accion 2011 - 2025 CPAA. Bogotá, Colombia.

18. República de Colombia. Ministerio de Comercio, Industria y Turismo. (13 de julio de 2012). Decreto 1500 de 2012. Por medio del cual se dictan medidas para la organización, articulación y funcionamiento del Sistema Administrativo Nacional de Competitividad e Innovación. Bogotá, Colombia.

19. República de Colombia. Presidencia de la República. (9 de Septiembre de 2016). ¿quienes somos?: Sistema Nacional de Competitividad, Ciencia, Tecnología e Innovación. Obtenido de Sistema Nacional de Competitividad, Ciencia, Tecnología e Innovación: http://www.colombiacompetitiva.gov.co/sncei/Paginas/quienes-somos.aspx

20. Santamaría, A. R. (Febrero de 2009). Consultoria Empresarial para el Sector Apícola. Diagnóstico productivo y comercial de la cadena apícola de los programas para la sustitución de cultivos ilícitos y desarrollo alternativo de Accón Social y UNODC. Bogotá, Colombia: Oficina de la Naciones Unidas contra la Droga y el Delito UNODC. Agencia Presidencial para la Acción social y la Cooperación Internacional Acción Social.

21. Vega Almeida, R. (2006). La relación dialógica entre la planificación estratégica y el aprendizaje organizacional. Acimed, 14(6).

22. World Economic Forum. (2011). The global Competitiveness Report 2011-2012. Geneva: SRO-Kundig. 